\title{
THE DISTRIBUTION OF THE CELLS OF ORIGIN OF CALLOSAL PROJECTIONS IN CAT VISUAL CORTEX ${ }^{1}$
}

\author{
MARK A. SEGRAVES ${ }^{2}$ AND ALAN C. ROSENQUIST ${ }^{3}$ \\ Department of Anatomy, University of Pennsylvania, Philadelphia, Pennsylvania 19104
}

Received August 18, 1981; Revised January 25, 1982; Accepted February 26, 1982

\begin{abstract}
The distribution of neurons projecting through the corpus callosum (callosal neurons) was examined in retinotopically defined areas of cat visual cortex. As many callosal neurons as possible were labeled in a single animal by surgically dividing the posterior two-thirds of the corpus callosum and exposing the cut ends of callosal axons to horseradish peroxidase. The distribution of callosal neurons within a visual field representation was related to standard electrophysiological maps as well as to recording sites marked by electrolytic lesions. Callosal neurons were found in every retinotopically defined cortical area. The portion of the visual field representation that contained callosal neurons increased progressively from the area $17 / 18$ border to area 19 , to areas 20 and 21 , and to the lateral suprasylvian visual areas. In area 17, the portion of the visual field representation containing callosal neurons extended from the vertical meridian out to a maximum of $10^{\circ}$ azimuth. In the posteromedial lateral suprasylvian visual area, callosal neurons were present in a region extending from the vertical meridian representation out to a representation of $60^{\circ}$ azimuth. Most callosal neurons were medium to large pyramids at the border of layers III and IV. A few layer IV stellates were among the callosal neurons of areas 17 and 18. In area 19 and even more so in the lateral suprasylvian visual areas, callosal neurons included pyramidal and fusiform-shaped cells in layers V and VI. The laminar distributions of callosal neurons in areas 20 and 21 were similar to those of area 19 and the lateral suprasylvian visual areas. The widespread distribution of callosal neurons in areas 20 and 21 and in the lateral suprasylvian visual areas suggests that the regions of peripheral visual field representation in cat cortex, as well as the representations of the vertical meridian, have access to the opposite cerebral hemisphere. This finding is significant in light of demonstrations of the importance of some of these cortical areas in the interhemispheric transfer of visual learning.
\end{abstract}

Early investigations of cat visual callosal connections demonstrated 3 primary sites of commissural termination (Hubel and Wiesel, 1965; Garey et al., 1968; Wilson, 1968; Heath and Jones, 1970, 1971). They included the area $17 / 18$ border, the lateral half of area 19 , and the lateral suprasylvian cortex. Subsequent investigations have, with some exceptions, concentrated on the connections of neurons along the vertical meridian representation at the area 17/18 border (e.g., Toyama et al., 1974; Innocenti

'This work was supported by National Institutes of Health Grants EY02654 and GM00281 and by a Grass Foundation fellowship award to M.A.S. We wish to thank Jeanne Levy for technical assistance as well as John and Bette Woolsey, Tom Sarrantonio, and Pauline Wong for preparation of the figures. We have presented preliminary reports of this study elsewhere (Segraves, 1979; Segraves and Rosenquist, 1980).

${ }^{2}$ Present address: Institute of Anatomy, Univeristy of Lausanne, Rue du Bugnon 9, 1011 Lausanne CHUV, Switzerland.

${ }^{3}$ To whom correspondence should be addressed at Department of Anatomy, University of Pennsylvania, 36th and Hamilton Walk, Philadelphia, PA 19104. and Fiore, 1976; Shatz, 1977b; Innocenti, 1980). Little is known about the callosal connections of the 6 electrophysiologically defined areas in the lateral suprasylvian cortex (Palmer et al., 1978) and of areas 20 and 21 (Heath and Jones, 1971; Tusa and Palmer, 1980). This is unfortunate since, in behavioral experiments, Berlucchi et al. (1979) showed that the callosal connections of the cat's suprasylvian cortex (including the lateral suprasylvian visual areas and area 21) make a significant contribution to the interhemispheric transfer of visual learning. By contrast, the callosal connections of areas 17,18 , and 19 are not necessary for cats to perform well in behavioral tests of interhemispheric transfer (Berlucchi et al., 1978).

In the present study, we have determined the distribution of neurons whose axons project through the corpus callosum (callosal cells or neurons) for all retinotopically defined visual cortical areas.

\section{Materials and Methods}

The two primary objectives of these experiments were (1) to examine the distribution of visual callosal neurons 
in all known retinotopic representations in the visual cortex and (2) to determine the laminar positions of cell bodies giving rise to callosal fibers. We labeled as many callosal neurons as possible in 5 cats by exposing the severed ends of the corpus callosum to horseradish peroxidase (HRP). In 2 of those animals, we preceded the labeling of callosal neurons with an electrophysiological recording experiment in which we placed electrolytic lesions at the representations of selected visual field loci.

The HRP labeling procedure was as follows: a cat was first anesthetized with sodium pentobarbital $(40 \mathrm{mg} / \mathrm{kg})$ and then given $0.2 \mathrm{mg}$ of atropine sulfate subcutaneously and 300,000 units of penicillin G intramuscularly. A large opening in the cranium was made, and the dura overlying one hemisphere was cut longitudinally just lateral to the superior sagittal sinus. Gentle retraction of one hemisphere was necessary to enable visualization of the corpus callosum. The posterior two-thirds of the callosum was divided by aspiration through an 18 gauge needle. Care was taken to avoid damaging the cortex on the medial surfaces of the cerebral hemispheres or the ependyma underlying the callosum. A cotton pledget saturated with $50 \%$ HRP (Boehringer Mannehim) or pieces of polyacrylamide gel impregnated with 15\% HRP (Griffin et al., 1979) were placed between the severed ends of the callosum. We found that the method of delivery had no apparent effect upon the resultant cell labeling. The HRP and its vehicle were left in place for a survival time of 48 $\mathrm{hr}$, during which the cat was alert and active. At the end of the survival period, the cat was anesthetized deeply with sodium pentobarbital and perfused with saline, followed by a solution of $0.5 \%$ paraformaldehyde and $2.5 \%$ glutaraldehyde in phosphate buffer $(\mathrm{pH} \mathrm{7.2)}$, and finally with a solution of $5 \%$ sucrose in phosphate buffer. The brain was blocked, removed from the cranium, and left overnight in $30 \%$ sucrose in water. Forty-eight-micrometer frozen sections were cut in the coronal plane. Every third section was stained for HRP using $o$-dianisidine as the chromogen (De Olmos, 1977). Every other section of the HRP-stained series was counterstained with thionin. Where necessary for identification of myeloarchitectonic bordeis, additional sections were stained for myelin using a Heidenhain protocol. The borders of areas 17, 18, and 19 were visible in myelin-stained sections. In these 3 areas, myeloarchitecturally defined borders correspond closely to electrophysiologically defined borders (Tusa et al., 1978, 1979). We determined the borders of areas 20 and 21 using the anatomical definition of these areas made by Heath and Jones $(1970,1971)$ and the electrophysiological definition of their borders made by Tusa and Palmer (1980). The lightly myelinated character of area 20 in comparison to the surrounding cortex and the relatively heavily myelinated appearance of area 21a were additional aids to the definition of borders for these areas. The borders of the lateral suprasylvian visual areas were not distinguishable in myelin-stained sections, and therefore, they were obtained by comparison to standard electrophysiological maps (Tusa et al., 1982).

A low magnification photomicrograph of a coronal section showing portions of left and right hemispheres with cut corpus callosum is provided in Figure 1. Dark $\mathrm{HRP} / o$-dianisidine reaction product is visible in callosal

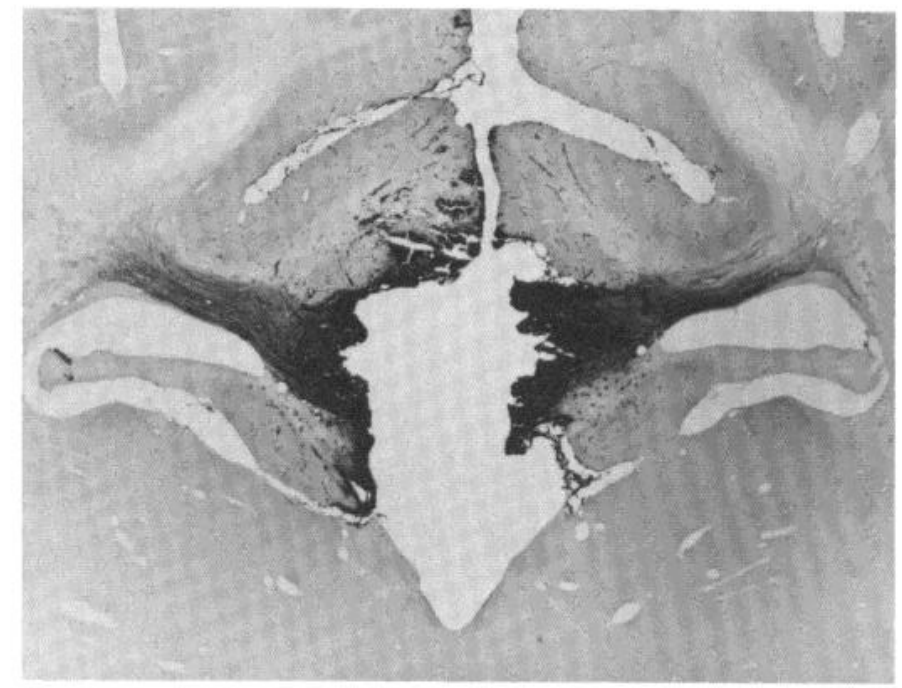

Figure 1. Unstained coronal section through the region of a callosal cut. This section is taken from same experiment illustrated in Figure 2. Dark HRP/o-dianisidine reaction product is visible in callosal axons. Damage to the cingulate gyrus is typical for the experiments included in this report.

axons extending from the cut. In this particular experiment, we retracted the left hemisphere during surgery. The resultant damage to the left cingulate gyrus typifies that found in other experiments from this series. In each case, we restricted our observations to the undamaged hemisphere.

In every cat, there was always some spread of HRP from its site of placement between the cut ends of the callosum to the adjacent cingulate gyri. This diffusion of HRP was sufficient to produce retrograde filling of neurons in the thalamus. In the animals used in this report, the thalamic label included a primary focus of HRP-filled neurons within the anterior nuclear group with additional light label in the ventral anterior, medial dorsal, reuniens, and lateral dorsal nuclei. The thalamic labeling produced was very similar to that reported by Niimi et al. (1978) and Robertson and Kaitz (1981) following injections of HRP in cingulate and retrosplenial cortex. Great care was taken to ascertain that there was no labeling of neurons in visual thalamic nuclei, including the dorsal lateral geniculate nucleus, the pulvinar, and the lateral posterior complex.

When electrophysiological recording preceded HRP labeling, our preparation and recording procedures were very similar to those employed by Tusa et al. (1978). In each experiment, electrode penetrations were made in area 17 and the posteromedial lateral suprasylvian area of a paralyzed, nitrous oxide-anesthetized cat. Electrolytic lesions (10 $\mu \mathrm{A}$ D.C. for $10 \mathrm{sec})$ were used to mark selected points in each visual field representation. At the end of the recording session, infusion of paralyzing agents was stopped and the cat was maintained on the respirator until capable of breathing on its own. Twelve hours later, the animal underwent the surgical and histological procedures described for HRP labeling.

To examine the laminar distribution of callosal neurons, criteria for delimiting laminae were taken from Otsuka and Hassler (1962) for areas 17, 18, and 19 and 
from Sanides and Hoffmann (1969) for the lateral suprasylvian visual areas. Cell counts were made in sections selected from the brains illustrated in Figures 2 and 3. The counts included all labeled neurons with the origin of one or more dendrites clearly visible. The number of neurons counted in each lamina was not corrected to account for variations in neuron diameter among laminae. Our primary intention was to compare the laminar distribution of callosal neurons among cortical areas. The effect of correcting cell counts in a single section (using Konigsmark's formula 4 in Nauta and Ebbesson, 1970) was to lower the percentage of labeled neurons in layer III and raise the percentage in layer VI but only by about $3 \%$ in both instances.

\section{Results}

Distribution of callosal neurons in retinotopically defined cortical areas. Exposing the severed ends of the corpus callosum to HRP resulted in the labeling of neurons in all retinotopically defined cortical areas (Figs. 2 and 3). The density of labeling varied, but there were relatively few regions of the cortex where labeling was absent. HRP-labeled cells were present in each of the 13 retinotopically defined cortical areas. Labeled neurons in area 17 combined with labeled neurons in area 18 to form a band along the entire area 17/18 border. Label at the area $17 / 18$ border is visible in every section illustrated in Figures 2 and 3 . In rostral coronal sections, the region of area 17 containing labeled neurons extended medially no further than the suprasplenial sulcus (Figs. 2, $A$ to $C$, and $3, A$ to $F$ ). At caudal anterior-posterior levels, the region of label did not extend more medially than the crown of the lateral gyrus (Figs. 2, $D$ to $I$, and $3, G$ to $I$ ). HRP labeling was completely absent from all portions of the peripheral visual ficld representation in area 17 on the medial surface of the hemisphere. HRP-labeled neurons in area 18 were present along the entire area $17 / 18$ border. The region of label in area 18 extended laterally from the area $17 / 18$ border to occupy about one-half of the cortex included in area 18. In some coronal sections, there was also a focus of label at the myeloarchitecturally defined area $18 / 19$ border (Figs. $2, D, G$, and $H$, and $3 E$ ). Patches of callosal neurons at the area 18/19 border were present at two locations in the experiment illustrated in Figure 2 and in one location in the experiment illustrated in Figure 3. These patches were suspected to be analogous to the patches of callosal terminals at the area 18/19 border described by Sanides and Albus (1980) and, if so, were located at positions along the area $18 / 19$ border where receptive field centers are within $5^{\circ}$ of the vertical meridian ('Tusa et al., 1979). The density and distribution of label in area 19 were variable. In coronal sections rostral to the posterior suprasylvian gyrus, HRP label was concentrated primarily in the lateral portion of area 19. At the level of the posterior suprasylvian gyrus, callosal neurons were present throughout most of area 19; the greatest concentration appeared to be at the border of area 19 with area 2 la (Fig. $2 F$ ). In all 6 areas of the lateral suprasylvian cortex (the anteromedial (AMLS) posteromedial (PMLS), ventral (VLS), anterolateral (ALLS), posterolateral (PLLS), and dorsal lateral suprasylvian (DLS) areas of Palmer et al., 1978), labeled neurons were present virtually everywhere (Figs. $2, A$ to $E$, and $3, A$ to $F$ ). For example, the high density of callosal neurons in area PMLS decreased only at a portion of PMLS near the outer edge of the middle suprasylvian sulcus (Fig. $2 \mathrm{C}$ ). On standard electrophysiological maps, this portion of area PMLS corresponds to a representation of the far peripheral horizontal meridian. The density of label in the ventral lateral suprasylvian area (VLS) was not as heavy as that found in other lateral suprasylvian areas. HRP-labeled neurons were found throughout areas 20a, 20b, 21a, and 21b (Figs. 2, $E$ to $I$, and $3, E$ to $H$ ).

Relationship of the location of callosal neurons to maps of visual cortex. Callosal neurons were clearly present in every retinotopically defined cortical area. However, comparing the position of label shown in Figures 2 and 3 with standard electrophysiological maps revealed that callosal neurons did not occupy the same amount of visual field representation in every cortical area.

We estimated the segment of an individual visual field representation that contained callosal neurons by comparing the distribution of labeled neurons in coronal sections to a standard series of coronal section drawings overlaid with retinotopic maps (Tusa et al., 1982). Callosal neurons were present in the segment of area 17 containing receptive field centers ranging from 0 to $5^{\circ}$ from the vertical meridian. In area 18 , callosal neurons were located in cortex representing the vertical meridian at the area $17 / 18$ border and extending out to 5 to $10^{\circ}$ azimuth. Callosal neurons in area 19 were present out to a maximum of 10 to $20^{\circ}$. In the lateral suprasylvian visual areas, the maximum extent of label ranged from 10 to $40^{\circ}$ from the vertical meridian, depending upon the anterior-posterior level at which a coronal section intercepted a visual field representation. The area of the visual field representation containing labeled neurons in areas 20a, 20b, 21a, and 21b was difficult to estimate because of the small size of these areas and the difficulty involved in determining their boundaries. Labeled neurons seemed to be present, in varying densities, in all portions of these 4 visual areas. Based upon the amount of visual field represented in these areas (Tusa and Palmer, 1980), we concluded that callosal neurons were present at points representing from the vertical meridian out to the far periphery in areas $20 \mathrm{a}, 20 \mathrm{~b}$, and $21 \mathrm{~b}$. Callosal neurons in area 21 a were at points representing up to $20^{\circ}$ azimuth, the maximal extent of the visual field representation in that area.

We obtained a more precise measurement of the segment of the visual field representation containing callosal neurons in areas 17 and PMLS by combining electrophysiological mapping with HRP labeling of callosal neurons. An experiment that used these techniques is illustrated in Figures 4 to 6 . Small electrolytic lesions were used to mark the recording sites of visual field loci, and, as in previous experiments, as many callosal cells as possible were labeled by placing HRP directly within a cut through the posterior two-thirds of the corpus callosum (Fig. 4). In Figures 5 and 6, the positions of HRPlabeled neurons and reconstructions of electrode penetrations in areas 17 and PMLS are shown on drawings of 


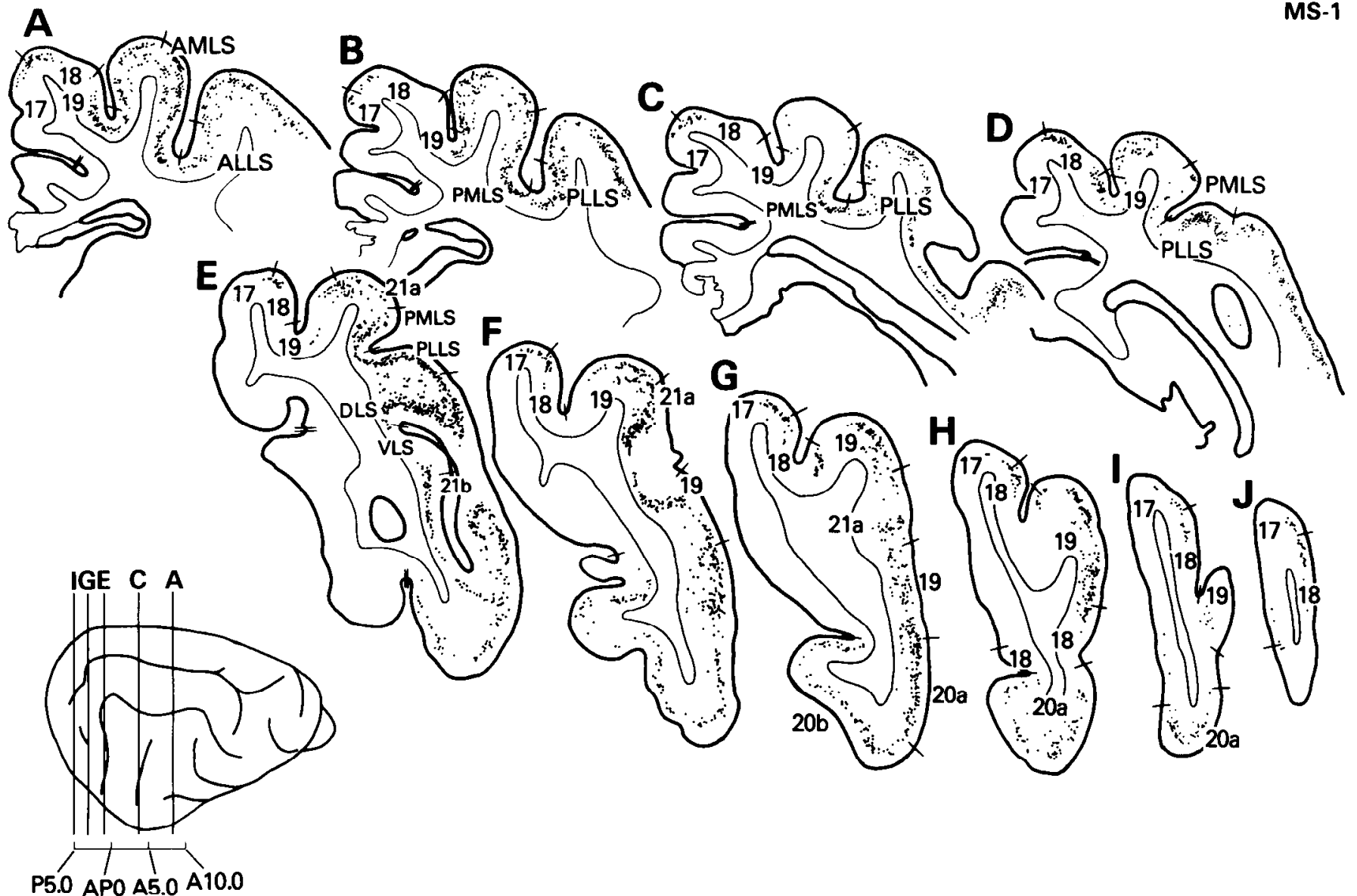

Figure 2. A series of coronal sections of the right hemisphere from a cat whose corpus callosum was divided and exposed to HRP. HPR-labeled cells are marked by dots. The single bars mark the boundaries between visual areas. HRP label in the cortex outside of the double bars in sections $A$ to $E$ is not shown. The posterior two-thirds of the corpus callosum was cut and a cotton pledget saturated with $50 \%$ HRP was placed between the cut ends of the callosum for a survival period of $48 \mathrm{hr}$. The tissue was reacted with o-dianisidine. Borders between areas were obtained form adjacent myelin-stained material or from standard electrophysiological maps when myeloarchitectural differences were not distinguishable. The names of the lateral suprasylvian areas of Palmer et al. (1978) - anteromedial (AMLS), posteromedial (PMLS), ventral (VLS), anterolateral (ALLS), posterolateral (PLLS), and dorsal lateral suprasylvian (DLS) areas-are used.

transverse sections through the lateral gyrus and the medial bank of the middle suprasylvian sulcus. Callosal neurons in area 17 were confined to a region where receptive fields ranged from those centered on the vertical meridian to fields centered at from 5 to $10^{\circ}$ azimuth (Fig. 5). It was also apparent in area 17 that, as electrode penetrations moved caudally closer and closer to the area centralis representation (going from $A$ to $B$ to $C$ in Fig. $5)$, the outer limit of the segment containing callosal neurons moved closer and closer to the vertical meridian representation. In area PMLS, virtually the entire visual field representation contained labeled neurons (Fig. 6). In the coronal section illustrated in Figure $6 A$, HRPlabeled neurons were present near a lesion marking a receptive field center of $60^{\circ}$ azimuth. The density of labeled neurons varied. Relatively few callosal neurons were present in regions of the cortex with receptive field centers 30 to $60^{\circ}$ from the vertical meridian. A high density of labeling was found in a region extending from the vertical meridian representation, at the fundus of the middle suprasylvian sulcus, out to the cortex containing neurons with receptive field centers of $20^{\circ}$ azimuth.
Laminar distribution of callosal neurons. We examined and compared the laminar distribution of callosal neurons in areas 17, 18, and 19 and lateral suprasylvian visual areas AMLS and PMLS. Figure 7 is a photomicrograph of HRP-labeled callosal neurons in area 18. Camera lucida drawings of the neuron labeling obtained in these experiments are provided in Figures 8 to 10 , and histograms of the laminar distributions of callosal neurons in areas 17, 18, 19, AMLS, and PMLS are provided in Figure 11.

The laminar distributions and morphologies of callosal neurons in areas 17 and 18 were similar. Most callosal neurons in areas 17 and 18 were medium to large pyramidal neurons, 10 to $18 \mu \mathrm{m}$ in diameter, located in lower layer III and upper layer IV. A few labeled stellate neurons, 10 to $14 \mu \mathrm{m}$ in diameter, were present in layer IV in both areas 17 and 18. There was a very small percentage of medium size pyramidal and fusiformshaped callosal neurons in layers V and VI of areas 17 and 18. In area $19,87 \%$ of the callosal neurons were located in layers III and IV, and the percentage of callosal neurons in layers V and VI increased from that found in 


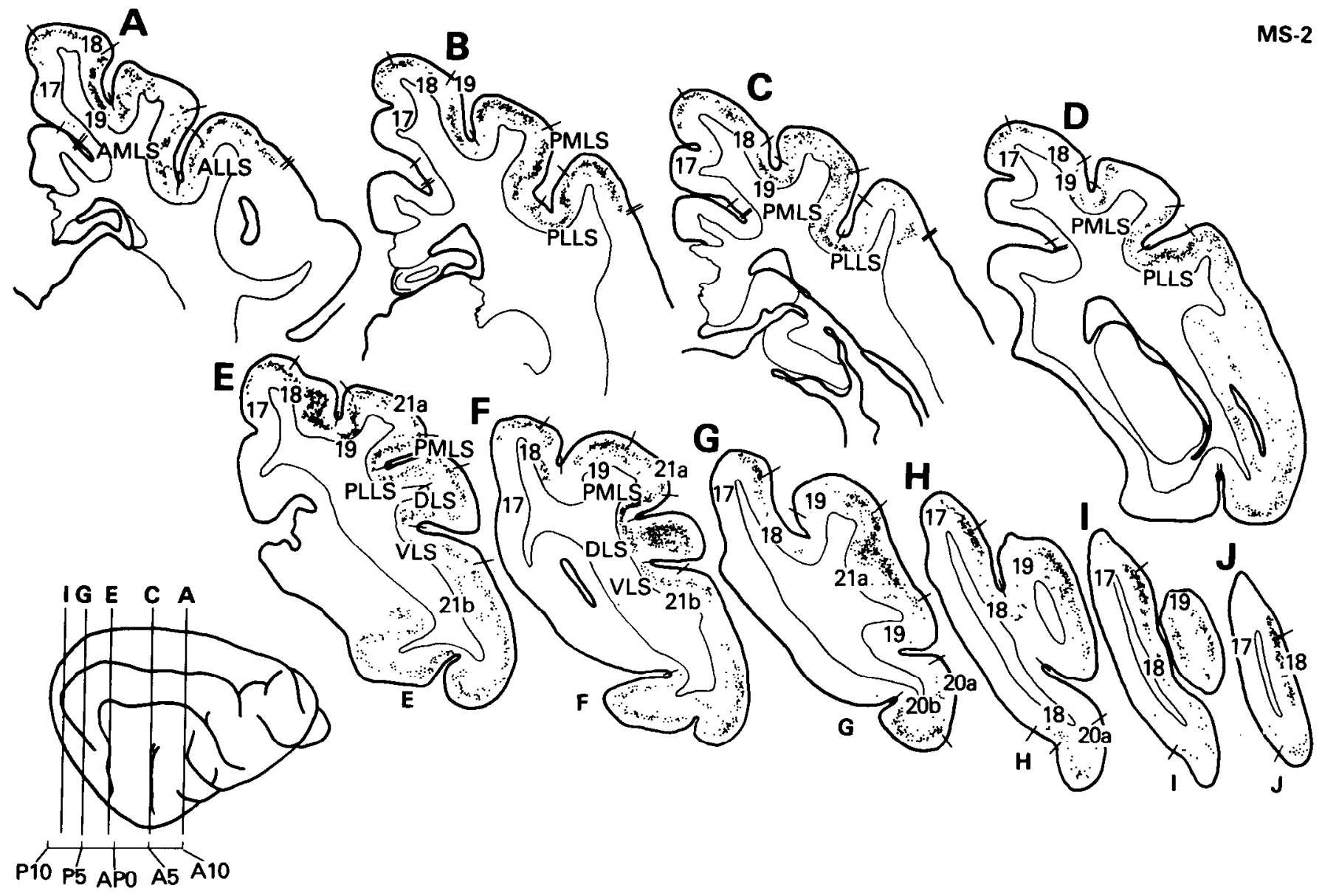

Figure 3. A series of coronal sections of the right hemisphere from a second callosal cut experiment. The same format and experimental procedure as for Figure 2 were used. HRP label in the cortex outside of the double bars in sections $A$ to $D$ is not shown.

areas 17 and 18 to roughly $13 \%$. In lateral suprasylvian areas AMLS and PMLS, the majority of callosal neurons were also pyramidal neurons in layers III and IV. However, the number of labeled pyramidal and fusiformshaped neurons in layers V and VI represented about $26 \%$ of the total number of callosal neurons. The laminar distributions of callosal neurons in areas 20 and 21 and in the remaining lateral suprasylvian visual areas resembled the distribution seen in areas 19, AMLS, and PMLS.

\section{Discussion}

Methodological considerations. Applying HRP directly to the transected axons of the corpus callosum was an effective means for labeling callosal neurons. However, this technique was accompanied by the danger of leakage to areas surrounding the corpus callosum. HRP reaction product was visible in the cingulate and retrosplenial cortex and was present in sufficient concentrations to produce labeling of neurons in thalamic nuclei that project to the cingulate and retrosplenial cortex (Niimi et al., 1978; Robertson and Kaitz, 1981). The thalamic label included a primary focus in the anterior nuclear group with additional light label in the ventral anterior, medial dorsal, reuniens, and lateral dorsal nuclei. Although a faint projection from the dorsal cap of the ventral anterior nucleus to the visual cortex exists (D. Raczkowski, personal communication), the presence of light ventral anterior labeling in these experiments is better explained by the diffusion of HRP to the limbic cortex. The leakage of HRP to the cingulate and retrosplenial cortex forced us to consider the possibility that this would cause retrograde filling of neurons in the visual cortex that project to the cingulate and retrosplenial cortex. Published reports describe no projections from the visual cortex to these limbic areas, and an examination of the ipsilateral cortico-cortical projections of the visual cortex in our own laboratory have not revealed such projections $(\mathrm{L}$. L. Symonds, personal communication). HRP-labeled neurons were never found in the dorsal lateral geniculate nucleus, the pulvinar, or the lateral posterior complex, indicating that HRP diffusing from the corpus callosum was not picked up directly by the visual cortex. If this had happened, it might have resulted in the labeling of neurons in the visual cortex with ipsilateral cortico-cortical projections. Moreover, if it were the case that neurons in the visual cortex with ipsilateral cortical projections were labeled, we would expect to have seen a significant number of labeled neurons in layer II (Gilbert and Kelly, 1975), and we did not. The distribution of callosal neurons demonstrated in this paper was corroborated by experiments described in the paper that follows (Segraves and Rosenquist, 1982), where small injections of HRP and $\left[{ }^{3} \mathrm{H}\right]$ leucine were made in the visual cortex.

Two additional considerations should be mentioned 


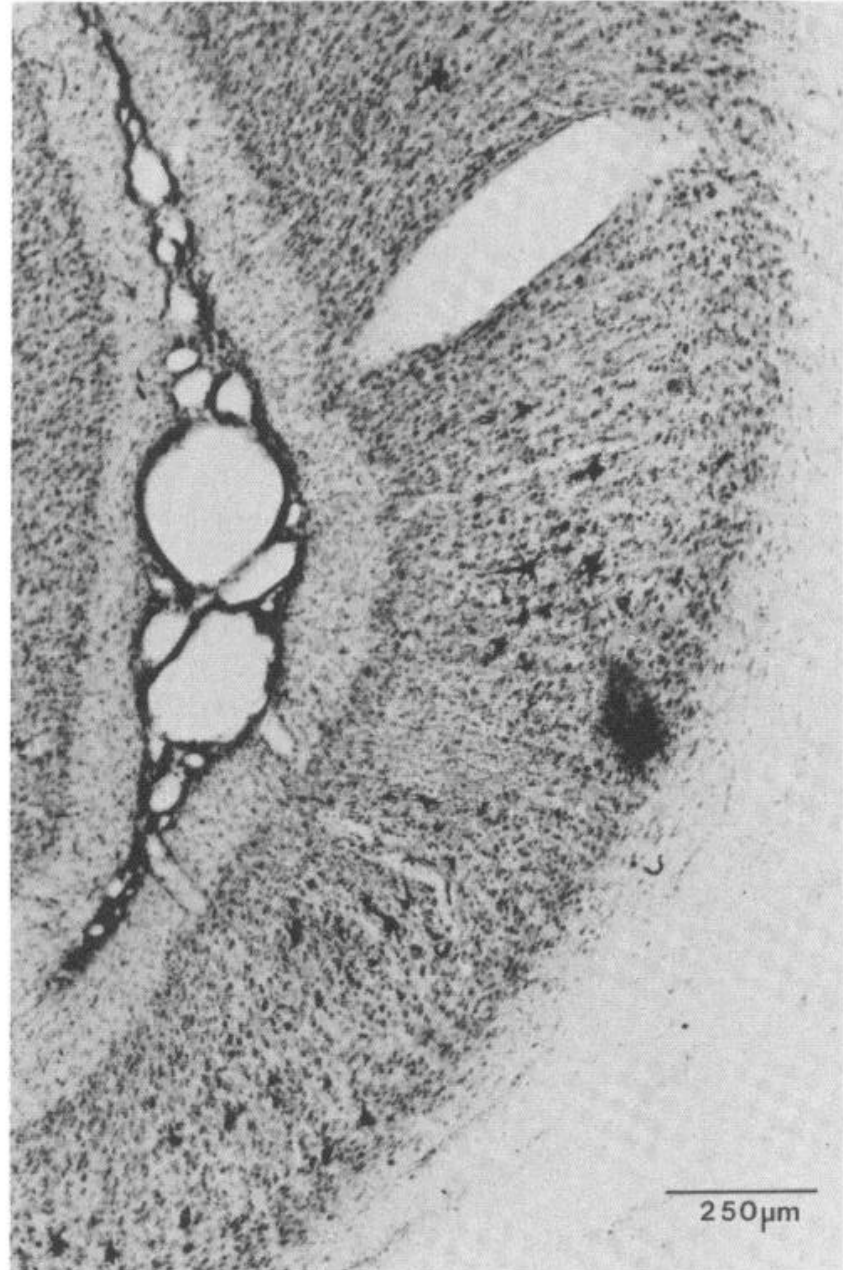

Figure 4. HRP-labeled neurons and an electrode penetration in the medial bank of the middle suprasylvian sulcus. The lesion marked the end of an electrode penetration through area PMLS. The receptive field center recorded at the lesion site was at a position of $5^{\circ}$ azimuth, $-10^{\circ}$ elevation. The lesion was made using $10 \mu \mathrm{A}$ for $10 \mathrm{sec}$. $\mathrm{HRP} / o$-dianisidine reaction and thionin counterstain also were used. The same experiment is illustrated in Figures 5 and 6.

here. First, our technique of cutting and labeling callosal axons excludes the demonstration of cortical neurons whose axons pass through the anterior commissure. However, most of these neurons appear to be located in nonvisual areas of the cortex (Jouandet, 1981). Second, the population of cells that we have labeled might include neurons that project subcortically. The following paper (Segraves and Rosenquist, 1982) demonstrates the extensive distribution of terminals of callosal neurons in the contralateral visual cortex. However, it is also possible that some of the cells that we have labeled terminate in subcortical sites, including, for example, the contralateral thalamus (Rinvik, 1968; Goldman, 1979) and the superior colliculus (Powell, 1976; Antonini et al., 1979).

Relationship of callosal neurons to maps of visual cortex. We have noted a progressive increase in the area of the visual field representation containing callosal neurons in areas 17,18, and 19, and in the lateral suprasylvian cortex. A similar progression also has been observed following lesions of the corpus callosum (Ebner and
Myers, 1965) and the lateral and posterolateral gyri (Sanides, 1978) and after multiple injections of HRP in the contralateral hemisphere (Payne et al., 1980).

The distribution of callosal neurons was very extensive in the lateral suprasylvian cortex and in both divisions of areas 20 and 21 . In these areas of the cortex, there were very few regions where callosal neurons were absent. Heath and Jones $(1970,1971)$, using cortical lesions, showed that degenerating commissural terminals were present in varying densities throughout portions of the lateral suprasylvian cortex corresponding to areas AMLS and PMLS and also throughout areas of the cortex equivalent to areas 20a, 20b, and 21a. Shatz (1977b) observed that HRP-labeled neurons in the lateral suprasylvian cortex projecting to the contralateral area $17 / 18$ border were not restricted to the vertical meridian representation. Recent anatomical studies (Sanides, 1978; Keller and Innocenti, 1981) have emphasized that callosal neurons and terminals are concentrated near representations of the vertical meridian and area centralis in areas AMLS, PMLS, and PLLS. We also see higher densities of callosal neurons near representations of the vertical meridian in these areas (e.g., Fig. 6). However, we are compelled not to overlook the presence of callosal neurons virtually throughout the 6 visuotopic representations in the lateral suprasylvian cortex, as well as in areas 20 and 21 (Figs. 2 and 3), in regions where receptive fields are centered some distance away from the vertical meridian. The receptive fields of cells in the peripheral visual field representations of the lateral suprasylvian visual areas and areas 20 and 21 are several times larger than the receptive fields at similar locations in areas 17, 18, and 19 (Palmer et al., 1978; Tusa et al., 1978, 1979; Tusa and Palmer, 1980), and the large receptive fields of callosal neurons in these regions may abut the vertical meridian (Berlucchi, 1981). However, this evidence does not conflict with the conclusion that peripheral portions of the visual field representations in these areas have access to the contralateral cortex.

Current electrophysiological evidence is ambiguous regarding the role of the corpus callosum in the transfer of information about peripheral visual fields. Direct recordings from the lateral suprasylvian visual areas have demonstrated that receptive fields commonly extend into the ipsilateral hemifield (Spear and Baumann, 1975; Palmer et al., 1978; Marzi et al., 1980). Palmer et al. (1978) have found receptive fields centered as much as $25^{\circ}$ into the ipsilateral hemifield. Marzi et al. (1980) found that the borders of receptive fields could extend up to $30^{\circ}$ into the ipsilateral hemifield and that the ipsilateral hemifield component of these receptive fields depended on the corpus callosum. In contrast, several studies of the responses of axons in the corpus callosum found that almost all receptive fields were less than $10^{\circ}$ in diameter and included the vertical meridian or were in its immediate vicinity (Berlucchi et al., 1967; Hubel and Wiesel, 1967; Shatz, 1977a). At present, the reason for this discrepancy is unknown.

In macaques, owl monkeys, and bushbabies, the distribution of degenerating terminals following section of the splenium of the corpus callosum has been used as a method for determining the boundaries of the visual cortical areas (Van Essen and Zeki, 1978; Newsome and 


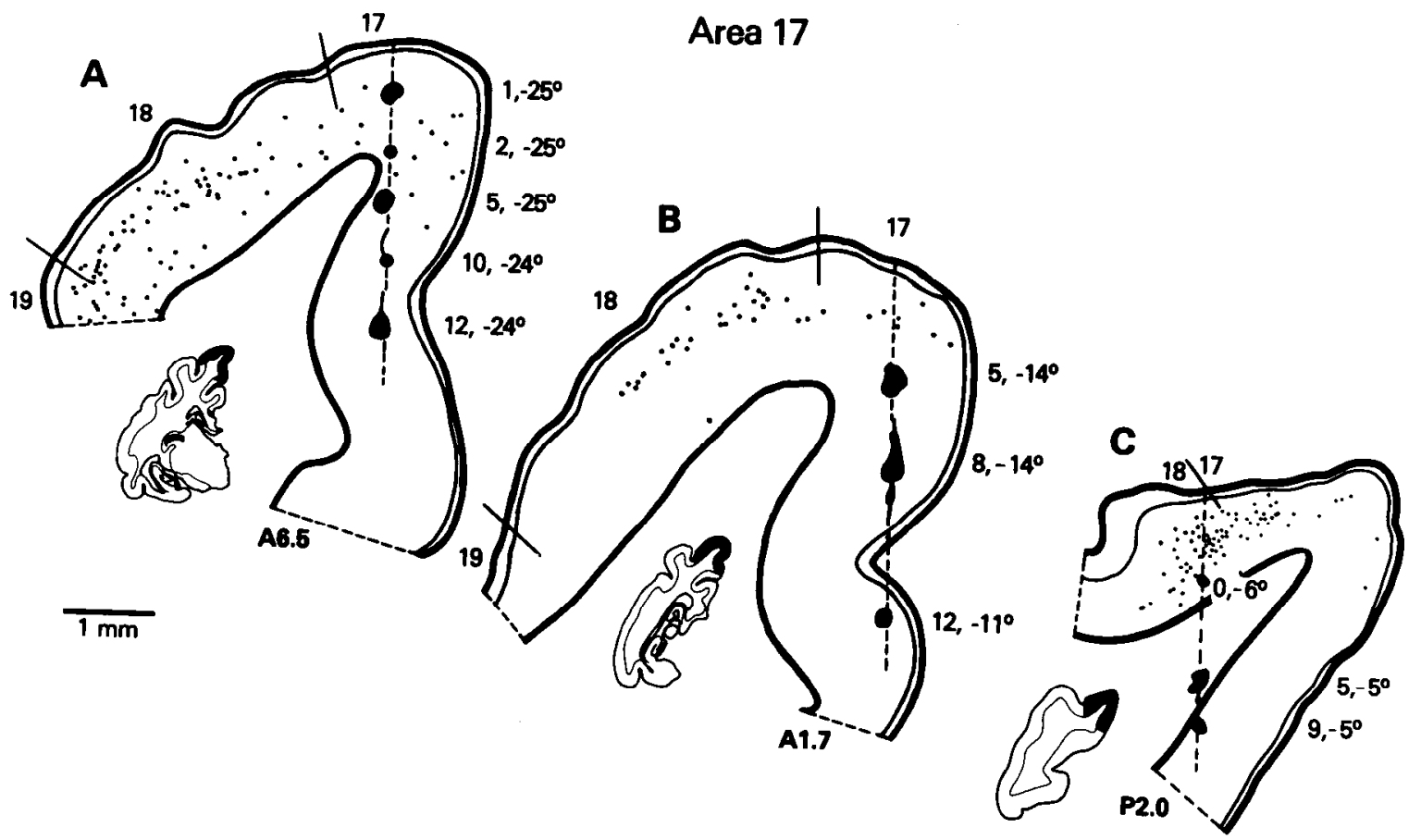

Figure 5. Position of HRP-labeled neurons and reconstructions of electrode penetrations in area 17. Transverse sections through cat lateral gyrus are shown. This is the same experiment as illustrated in Figures 4 and 6 . Small electrolytic lesions marked the recording sites of a number of visual field loci. As many callosal cells as possible have been labeled by placing HRP directly within a cut through the posterior two-thirds of the corpus callosum. Callosal cells are marked with small black dots. The visual field coordinates are listed in the form-azimuth, elevation-to the right of each lesion. In area 17 , callosal cells occupy a region extending from the vertical meridian representation at the area $17 / 18$ border out to no more than 5 to $10^{\circ}$ azimuth. Moving caudally, toward the representation of the area centralis (from $A$ to $B$ to $C$ ), the cells move progressively closer to the vertical meridian representation at the area $17 / 18$ border.

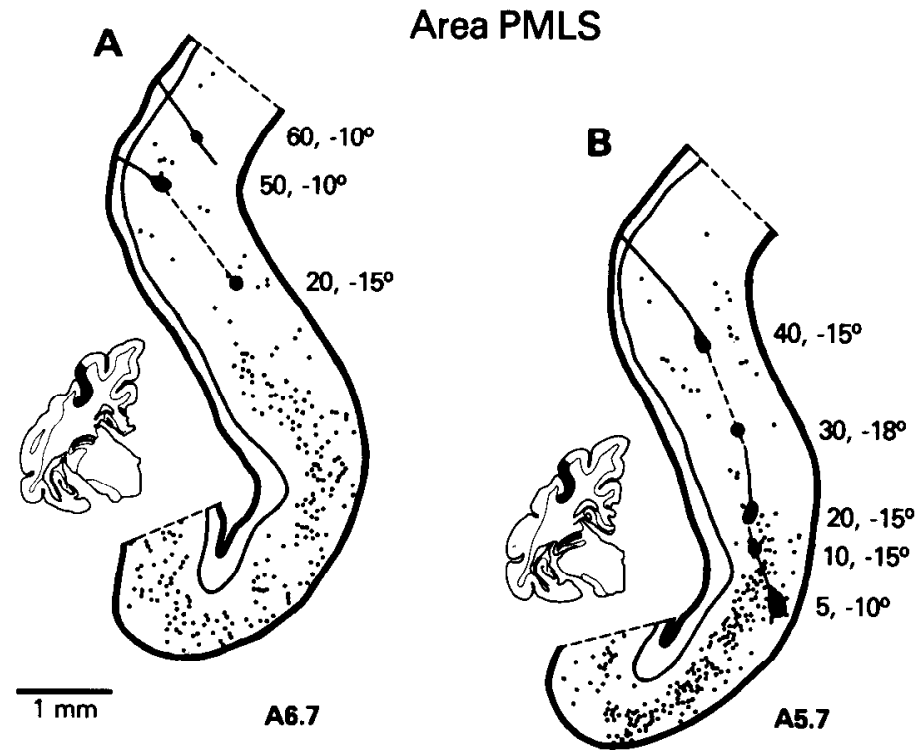

Figure 6. Positions of HRP-labeled neurons and reconstructions of electrode penetrations in area PMLS. These are transverse sections through the medial bank of the middle suprasylvian sulcus in the same experiment shown in Figures 4 and 5. In lateral suprasylvian visual area PMLS, callosal cells are located in all portions of the visual field representation from which we recorded. Note the callosal cells in section $A$ near a lesion marking a recording site $60^{\circ}$ from the vertical meridian. In both sections, there is a sharp increase in the density of cells beginning at about $20^{\circ}$ azimuth and extending to the vertical meridian representation at the fundus of the middle suprasylvian sulcus.
Allman, 1980). In these primates, the position of callosal degeneration appears to be a reliable marker for the vertical meridian representation at the area V1/V2 border as well as for several other vertical meridian representations in the extrastriate cortex. Unfortunately, this approach is not valid for marking borders in cat visual cortex. Although we have examined the distribution of callosal neurons and not terminals, the following paper (Segraves and Rosenquist, 1982) demonstrates that their distributions are approximately the same. The positions of callosal neurons at the area $17 / 18$ border are sufficiently well confined to serve as an adequate landmark for this border, but in all other cortical visual areas that we have examined, callosal cells are rather widely distributed, and although there may be an increased density of cells near a vertical meridian representation (as demonstrated in area PMLS; Fig. 6), it is not sufficient to localize the border of the visual area accurately.

Laminar distribution of callosal neurons. We have observed an increasing percentage of callosal neurons in layers $\mathrm{V}$ and VI as one moves laterally from the area $17 / 18$ border $(1 \%)$ to area $19(13 \%)$ to areas AMIS and PMLS (26\%). Keller and Innocenti (1981) have reported a similar pattern. This organization is analogous to that seen by Gilbert and Kelly (1975) for ipsilateral corticocortical projections. In their experiments, the percentage of layer VI neurons with terminations in the ipsilateral cortex was very low in areas 17 and 18 where a high percentage of layer VI neurons projected to the lateral geniculate nucleus. The percentage of layer VI neurons with ipsilateral cortico-cortical projections increased in 


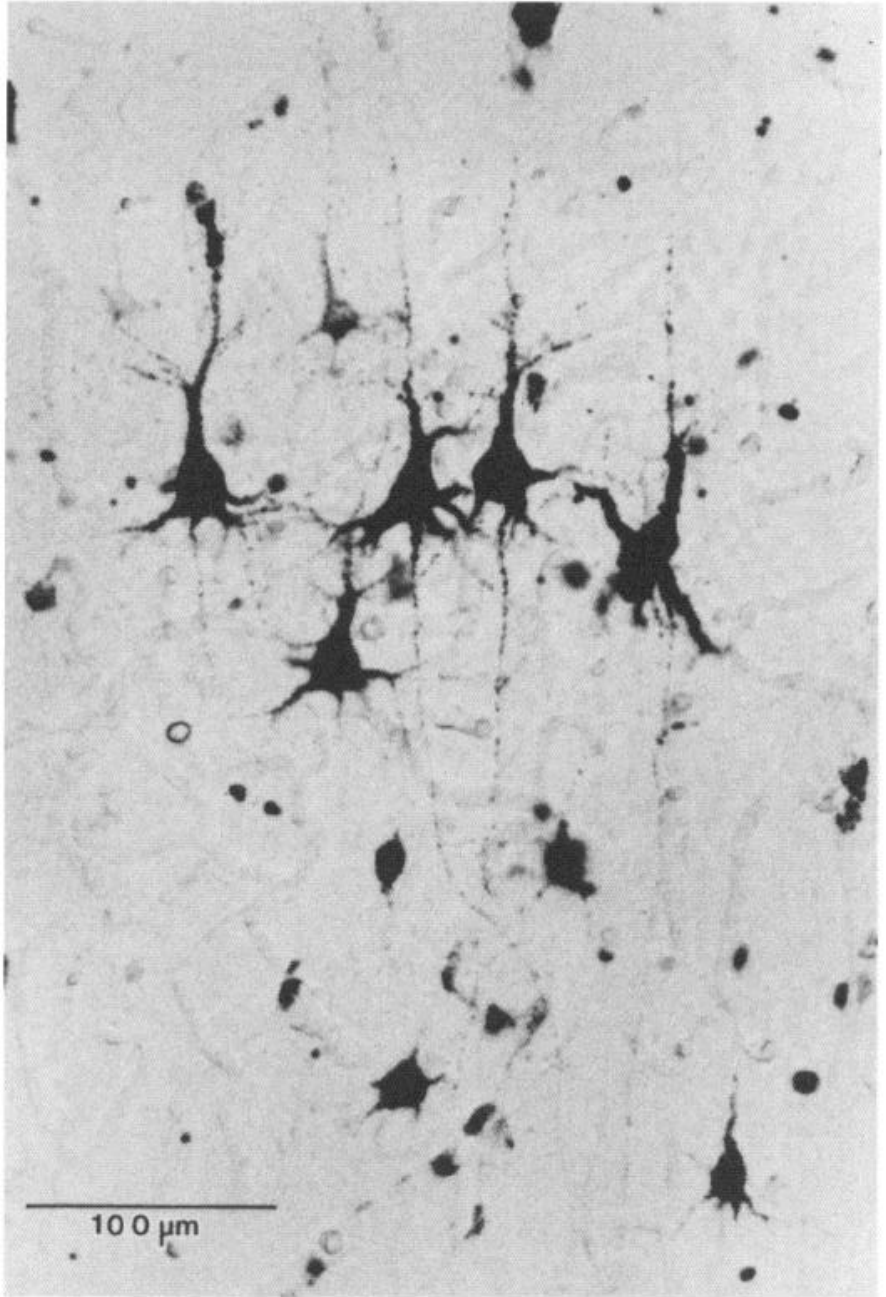

Figure 7. Photomicrograph of HRP-labeled callosal neurons in area 18. This corresponds to the area within the rectangle in Figure 8.

area 19 and increased further in the lateral suprasylvian cortex where very few corticogeniculate neurons were found.

Possible functional significance of callosal neurons. Two functional roles proposed for visual callosal neurons are binocular depth perception (Mitchell and Blakemore, 1970 ) and the interhemispheric transfer of visual learning (Myers, 1956; Sperry et al., 1956).

The role of the corpus callosum in binocular depth perception and the initiation of vergence eye movements is probably restricted to stimuli with relatively large disparities presented along the vertical meridian (Mitchell and Blakemore, 1970). Callosal neurons that might contribute to this form of depth perception are present in each of the 13 visual areas. Presently, there is no evidence to indicate that one visual area plays a more important role than another in this function.

Several behavior studies have examined the relationship of a number of visual cortical areas to the interhemispheric transfer of pattern discriminations in cats with split chiasms (Berlucchi et al., 1978, 1979; Turcotte et al., 1978). Unilateral or bilateral lesions of areas 17, 18, and 19 do not seem to affect interhemispheric transfer (Berlucchi et al., 1978). In contrast, marked effects have been demonstrated in cats with a unilateral lesion of the suprasylvian cortex, including areas 7 and 21 and portions of area 19 and the lateral suprasylvian visual areas (Berlucchi et al., 1979). A smaller unilateral lesion of a region corresponding to electrophysiologically defined areas AMLS and PMLS also resulted in poor interhemispheric transfer without producing the visual learning deficit associated with larger lesions. The transfer must occur during the discrimination learning, since cutting the corpus callosum after a pattern discrimination is learned by one hemisphere does not affect the savings in learning of the discrimination by the opposite hemisphere (Berlucchi et al., 1979). The results of these behavior studies help to underline the importance of the commissural connections of area 21 and the lateral suprasylvian visual areas. We have shown that these areas contain callosal neurons distributed throughout all portions of their visual field representations. Together, these results suggest that the callosal neurons of these areas provide the contralateral non-seeing hemisphere with a direct copy of the visual information transmitted to the seeing hem-

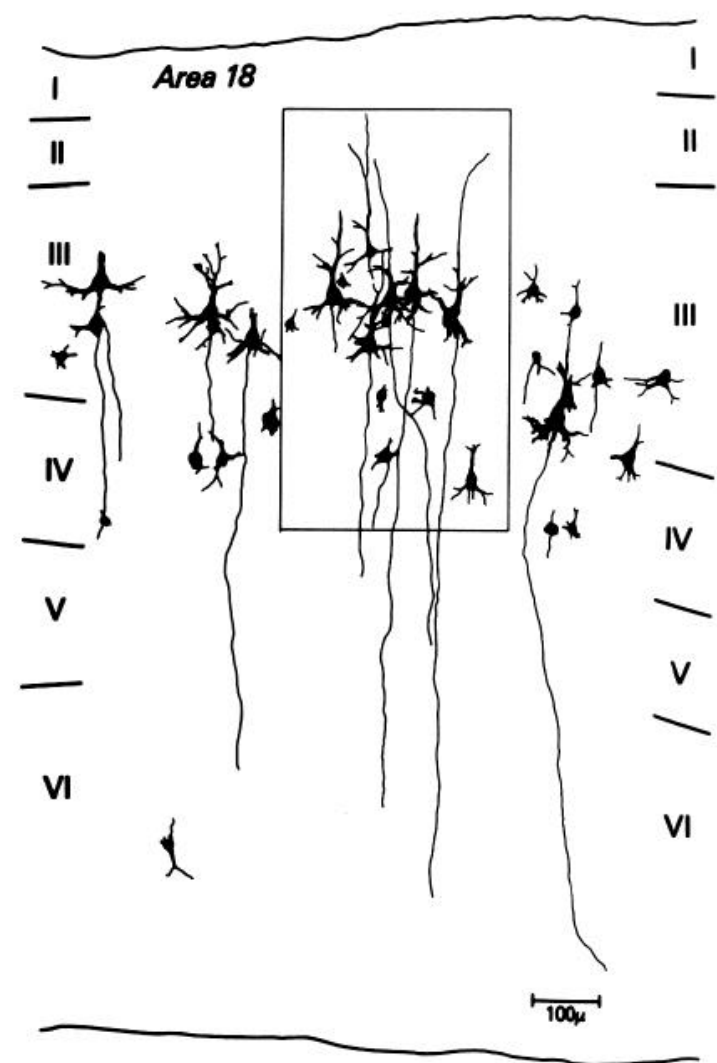

Figure 8. Camera lucida drawing of HRP-labeled callosal neurons in area 18 near the area $17 / 18$ border. The cells are primarily medium to large pyramids but include a few stellates. All cells are concentrated in lower layer III and upper layer IV with the exception of a single inverted pyramid in layer VI. A photomicrograph of the region within the rectangle is provided in Figure 7. This is from the same experiment as illustrated in Figure 3 . 


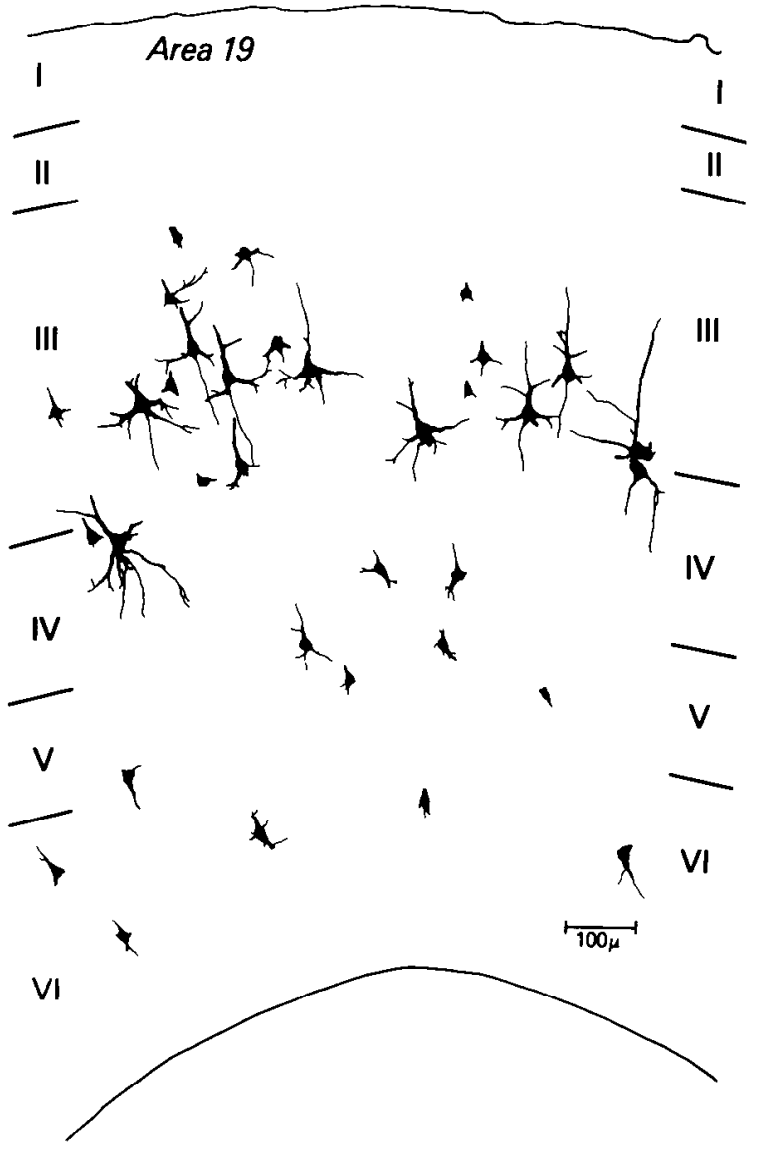

Figure 9. Camera lucida drawing of HRP-labeled callosal neurons in area 19. Most labeled neurons are medium to large pyramids in lower layer III and upper layer IV. Note the increase in the number of labeled pyramidal and fusiformshaped cells in layer V and VI above that present near the area $17 / 18$ border (Fig. 8). This is from the experiment as illustrated in Figure 2.

isphere during the discrimination trials. It is possible that the callosal connections of these areas perform a function similar to the role proposed by Seacord et al. (1979) for the callosal and anterior commissure connections of the monkey's inferotemporal cortex. They have suggested that interhemispheric transfer is an integral component of the pattern discrimination learning process that provides a means to attain "perceptual equivalence for patterns in left and right hemispheres."

Our results have shown that the traditional rule restricting callosal connections to representations of the vertical meridian is clearly not valid for all visual areas. It is true for areas 17, 18, and 19 but progressively less so for each. In contrast, callosal axons originating in areas 20 and 21 , and in the lateral suprasylvian visual areas transfer information from all portions of the visual field. Some of these same areas of the cortex are known to be important in interhemispheric transfer of visual learning. In the following paper (Segraves and Rosenquist, 1982), we will describe the connections of individual visual cortical areas with their counterparts and other areas in the opposite hemisphere.

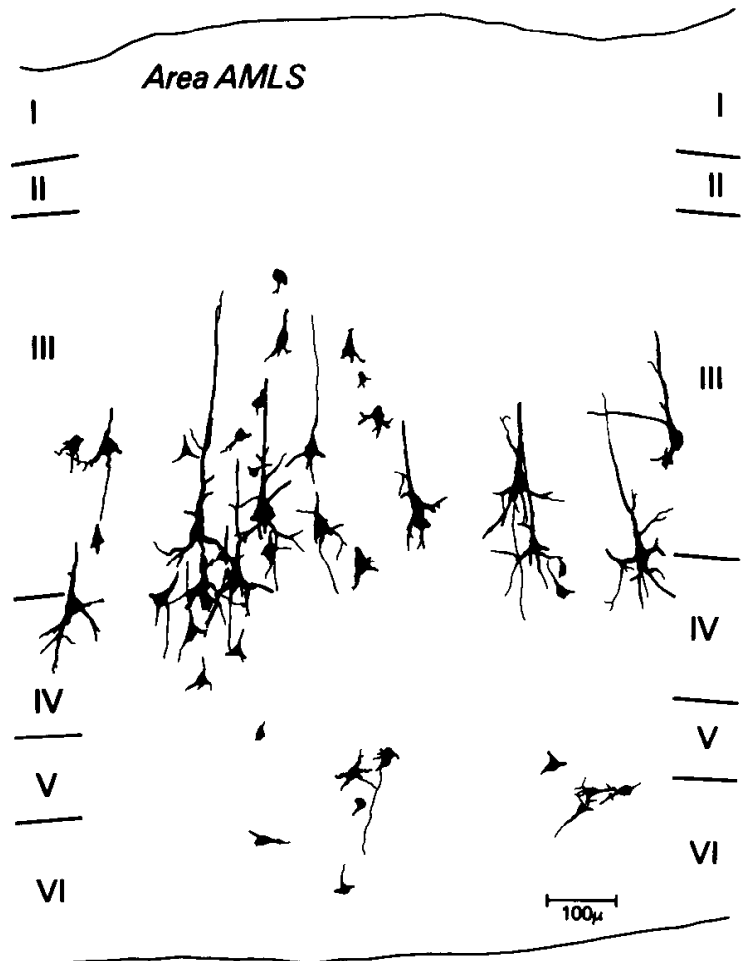

Figure 10. Camera lucida drawing of HRP-labeled neurons in lateral suprasylvian visual area AMLS. As was found at the area $17 / 18$ border and in area 19, most labeled cells are pyramidal neurons in lower layer III and upper layer IV, but there are also a significant number of pyramidal and fusiform-shaped cells in layers V and VI. This is from the same experiment as illustrated in Figure 3.

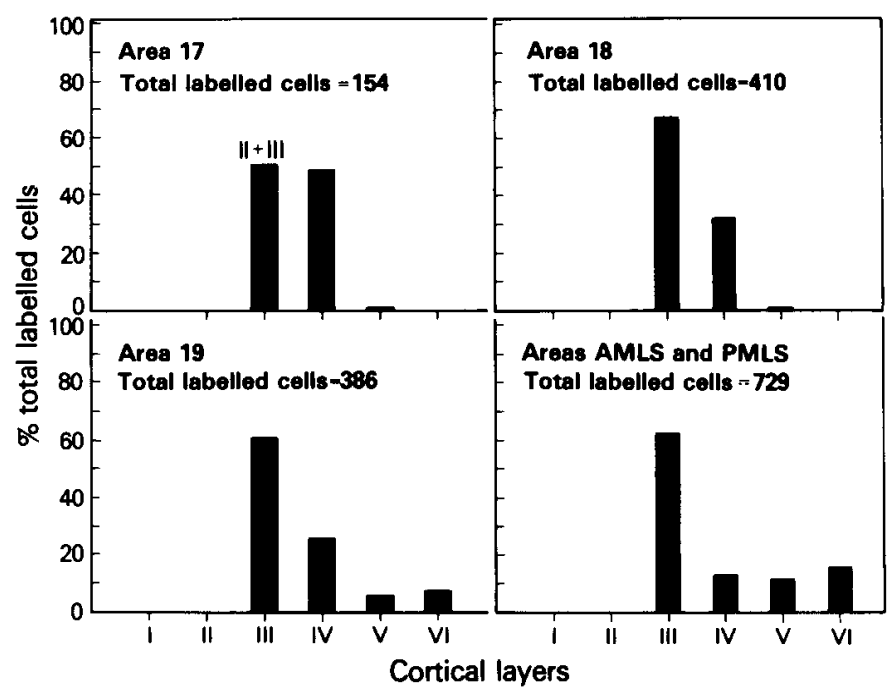

Figure 11. Hislograms showing the laminar distrubitons of labeled callosal cells in areas 17, 18, 19, AMLS, and PMLS. Layers II and III are combined for area 17 , since a division between these two layers is not distinguished easily. However, all cells in the layer II/III group were located in the lower half, near the layer III/IV border. No labeled cells were present in layer I of area 17, and no labeled cells were present in layers I or II in areas 18, 19, AMLS, or PMLS. Less than $0.5 \%$ of the total number of labeled cells were present in layer VI in areas 17 and 18 . Cells were counted in sections selected from the brains illustrated in Figures 2 and 3 . 


\section{References}

Antonini, A., G. Berlucchi, C. A. Marzi, and J. M. Sprague (1979) Importance of corpus callosum for visual receptive fields of single neurons in cat superior colliculus. J. Neurophysiol. 42: 137-152.

Berlucchi, G. (1981) Recent advances in the analysis of the neural substrates of interhemispheric communication. In Brain Mechanisms and Perceptual Awareness, O. Pomepeiano and C. A. Marsan, eds., pp. 133-152, Raven Press, New York.

Berlucchi, G., M. S. Gazzaniga, and G. Rizzolatti (1967) Microelectrode analysis of transfer of visual information by the corpus callosum. Arch. Ital. Biol. 105: 583-596.

Berlucchi, G., J. M. Sprague, F. Lepore, and G. G. Mascetti (1978) Effects of lesions of areas 17, 18 and 19 on interocular transfer of pattern discrimination in split-chiasm cats. Exp. Brain Res. 31: 275-297.

Berlucchi, G., J. M. Sprague, A. Antonini, and A. Simoni (1979) Learning and interhemispheric transfer of visual pattern discriminations following unilateral suprasylvian lesions in splitchiasm cats. Exp. Brain Res. 34: 551-574.

De Olmos, J. S. (1977) An improved HRP method for the study of central nervous connections. Exp. Brain Res. 29: 541-551.

Ebner, F. F., and R. E. Myers (1965) Distribution of corpus callosum and anterior commissure in cat and raccoon. J. Comp. Neurol. 124: 353-366.

Garey, L. J., E. G. Jones, and T. P. S. Powell (1968) Interrelationships of striate and extrastriate cortex with the primary relay sites of the visual pathway. J. Neurol. Neurosurg. Psychiatry 31: 135-157.

Gilbert, C. D., and J. P. Kelly (1975) The projections of cells in different layers of the cat's visual cortex. J. Comp. Neurol. 163: 81-106.

Goldman, P. S. (1979) Contralateral projections to the dorsal thalamus from frontal association cortex in the rhesus monkey. Brain Res. 166: 166-171.

Griffin, G., L. R. Watkins, and D. J. Mayer (1979) HRP pellets and slow-release gels: Two new techniques for greater localization and sensitivity. Brain Res. 168: 595-601.

Heath, C. J., and E. G. Jones (1970) Connections of area 19 and the lateral suprasylvian area of the visual cortex of the cat. Brain Res. 19: 302-305.

Heath, C. J., and E. G. Jones (1971) The anatomical organization of the suprasylvian gyrus of the cat. Ergeb. Anat. Entwicklungsgesch. 45: 4-64.

Hubel, D. H., and T. N. Wiesel (1965) Receptive fields and functional architecture in two nonstriate visual areas $(18$ \& 19) of the cat. J. Neurophysiol. 28: 229-289.

Hubel, D. H., and T. N. Wiesel (1967) Cortical and callosal connections concerned with the vertical meridian of visual fields in the cat. J. Neurophysiol. 30: 1561-1573.

Innocenti, G. M. (1980) The primary visual pathway through the corpus callosum: Morphological and functional aspects in the cat. Arch. Ital. Biol. 118: 124-188.

Innocenti, G. M., and L. Fiore (1976) Morphological correlates of visual field transformation in the corpus callosum. Neurosci. Lett. 2: 245-252.

Jouandet, M. (1981) Cortical field of origin of the cat's anterior commissure. Soc. Neurosci. Abstr. 7: 82.

Keller, G., and G. M. Innocenti (1981) Callosal connections of suprasylvian visual areas in the cat. Neuroscience, 6: 703712 .

Marzi, C. A., A. Antonini, M. DiStefano, and C. R. Legg (1980) Callosum-dependent binocular interactions in the lateral suprasylvian area of Siamese cats which lack binocular neurons in areas 17 and 18. Brain Res. 197: 230-235.

Mitchell, D. E., and C. Blakermore (1970) Binocular depth perception and the corpus callosum. Vision Res. 10: 49-54.

Myers, R. E. (1956) Function of the corpus callosum in interocular transfer. Brain 79: 358-363.

Nauta, W. J. H., and S. O. E. Ebbesson (1970) Contemporary Research Methods in Neuroanatomy, pp. 315-340, SpringerVerlag, New York.

Newsome, W. T., and J. M. Allman (1980) Interhemispheric connections of visual cortex in the owl monkey and the bushbaby. J. Comp. Neurol. 194: 209-233.

Niimi, K., M. Niimi, and Y. Okada (1978) Thalamic afferents to the limbic cortex in the cat studied with the method of retrograde axonal transport of HRP. Brain Res. 145: 225-238.

Otsuka, R., and R. Hassler (1962) Uber Aufbau and Gliederung der corticalen Sehesphare bei der Katze. Arch. Psychiatr. Nervenkr. 203: 213-234.

Palmer, L. A., A. C. Rosenquist, and R. J. Tusa (1978) The retinotopic organization of lateral suprasylvian visual areas in the cat. J. Comp. Neurol. 177: 237-256.

Payne, B. R., M. Harris-Moss, N. Berman, and E. H. Murphy (1980) Interhemispheric connections of the cat's visual cortex. Invest. Ophthalmol. Vis. Sci. Abstr. 1.

Powell, T. P. S. (1976) Bilateral cortico-tectal projection from the visual cortex in the cat. Nature 260: 526-527.

Rinvik, E. (1968) The corticothalamic projection from the gyrus proreus and the medial wall of the rostral hemisphere in the cat. An experimental study with silver impregnation methods. Exp. Brain Res. 5: 129-152.

Robertson, R. T., and S. S. Kaitz (1981) Thalamic connections with limbic cortex. I. Thalamocortical projections. J. Comp. Neurol. 195: 501-526.

Sanides, D. (1978) The retinotopic distribution of visual callosal projections in the suprasylvian visual areas compared to the classical visual areas $(17,18,19)$ in the cat. Exp. Brain Res. 33: 435-444.

Sanides, D., and K. Albus (1980) The distribution of interhemispheric projections in area 18 of the cat: Coincidence with discontinuities of the representation of the visual field in the second visual area (V2). Exp. Brain Res. 38: 237-240.

Sanides, F., and J. Hoffmann (1969) Cyto- and myeloarchitecture of the visual cortex of the cat and of surrounding integration cortices. J. Hirnforsch. 11: 79-104.

Seacord, L., C. G. Gross, and M. Mishkin (1979) Role of inferior temporal cortex in interhemispheric transfer. Brain Res. 167: 259-272.

Segraves, M. A. (1979) Interhemispheric connections of retinotopically defined visual cortical areas in the cat. Soc. Neurosci. Abstr. 5: 807.

Segraves, M. A., and A. C. Rosenquist (1980) The location of callosal projection neurons in relationship to maps of the visual field in cat cortex. Soc. Neurosci. Abstr. 6: 673 .

Segraves, M. A., and A. C. Rosenquist (1982) The afferent and efferent callosal connections of retinotopically defined areas in cat cortex. J. Neurosci. 2: 1090-1107.

Shatz, C. J. (1977a) Abnormal interhemispheric connections in the visual system of Boston Siamese cats: A physiological study. J. Comp. Neurol. 171: 229-246.

Shatz, C. J. (1977b) Anatomy of interhemispheric connections in the visual system of Boston Siamese and ordinary cats. $J$. Comp. Neurol. 173: 497-518.

Spear, P. D., and 'I. P. Baumann (1975) Receptive field characteristics of single neurons in lateral suprasylvian visual area of the cat. J. Neurophysiol. 38: 1403-1420.

Sperry, R. W., J. Stamm, and N. Miner (1956) Relearning tests for interocular transfer following divisions of optic chiasma and corpus callosum in cats. J. Comp. Physiol. Psychol. 49: 529-533.

Toyama, K., K. Matsunami, T. Ohno, and S. Tokashiki (1974) An intracellular study of neuronal organization in the visual 
cortex. Fxp. Brain. Res. 21: 45-66.

Turcotte, M., M. Ptito, M. C. Lassonde, and F. Lepore (1978) Effects of unilateral and bilateral lesions of the suprasylvian area on interocular transfer in the cat. Soc. Neurosci. Abstr. 4: 648 .

Tusa, R. J., and L. A. Palmer (1980) Retinotopic organization of areas 20 and 21 in the cat. J. Comp. Neurol. 193: 147-164.

Tusa, R. J., L. A. Palmer, and A. C. Rosenquist (1978) The retinotopic organization of area 17 (striate cortex) in the cat. J. Comp. Neurol. 177: 213-236.

Tusa, R. J., A. C. Rosenquist, and L. A. Palmer (1979) Retino- topic organization of areas 18 and 19. J. Comp. Neurol. 185: 657-678.

Tusa, R. J., L. A. Palmer, and A. C. Rosenquist (1981) Multiple cortical visual areas: Visual field topography in the cat. In Cortical Sensory Organization, Vol. 2, C. N. Woolsey, ed., pp. 1-31, Humana Press, Clifton, NJ.

Van Essen, D. C., and S. M. Zeki (1978) The topographic organization of rhesus monkey prestriate cortex. J. Physiol. (Lond.) 277: 193-266.

Wilson, M. E. (1968) Cortico-cortical connections of the cat visual areas. J. Anat. 102: 375-386. 